\title{
Transformation Texture in Deformed Stainless Steel
}

\author{
Saurabh Kundu and H. K. D. H. Bhadeshia \\ University of Cambridge \\ Materials Science and Metallurgy \\ Pembroke Street, Cambridge CB2 3QZ, U. K. \\ email:sk459@cam.ac.uk,hkdb@cam.ac.uk,url:www.msm.cam.ac.uk/phase-trans
}

In an elegant experimental study on an austenitic stainless steel, Gey et al. [1] found that not all of the 24 possible crystallographic variants of martensite $\left(\alpha^{\prime}\right)$ formed when the alloy underwent transformation during tensile deformation. The selection of particular variants was demonstrated by comparing calculated pole figures containing poles from all $24 \alpha^{\prime}$ orientations against measured pole-figures.

This variant selection was particularly noticeable in austenite $(\gamma)$ grains belonging to the Goss and Cube components of texture, which transformed to smaller fractions of martensite. This is presumably because the early stages of transformation are dominated by the $\alpha^{\prime}$ variants most favoured by the external stress.

The selection process was explained qualitatively by associating the martensite with the slip systems that have the greatest Schmidt factors, although even on the "activated" $\{111\}_{\gamma}$ planes, only a fraction of the possible variants were found.

We show in this note that the observed transformation textures can be calculated by considering the interaction of the applied stress with the martensite plates, based on a self-consistent set of crystallographic parameters.

It is often assumed in texture analysis that the orientation relationship between the austenite and martensite is that due to Kurdjumov-Sachs or Nishiyama-Wasserman [2-5], but it has been known for some time that the true relation must be irrational [6-9]. Although the difference between this irrational and assumed orientation may seem less than a few degrees, it is vital because the assumed orientations do not in general lead to an invariant-line between the parent and product lattices. The existence of an invariant line is an essential requirement for martensitic transformation to occur. It is not surprising therefore, that Nolze [10] in his experimental study of several hundred thousand $\gamma / \alpha$ orientation relations, found detailed deviations from assumed Kurdjumov-Sachs etc. orientations.

When considering martensitic transformation, the crystallographic set includes the habit plane and shape deformation apart from the orientation relation. This set is mathematically linked and should be considered as a whole in texture analysis [6-8]. Although the full set of necessary data do not exist for martensite in the 304 stainless steel of the type studied by Gey et al., there are crude habit 
plane data which can help select a self-consistent set [11].

The crystallographic set associated with a single martensite variant, gives a habit plane close to the approximately $\left\{\begin{array}{lll}2 & 5 & 9\end{array}\right\}_{\gamma}$ plane reported by Lagneborg [11]. The lattice parameter of austenite in 304 stainless steel is estimated to be $0.3589 \mathrm{~nm} \mathrm{[12]} \mathrm{and} \mathrm{that} \mathrm{of} \mathrm{the} \mathrm{martensite} \mathrm{is} \mathrm{taken} \mathrm{to} \mathrm{be}$ $0.2873 \mathrm{~nm}$. This standard set is given as follows, calculated as in [9]:

$$
\begin{aligned}
& \text { Habit plane } \quad(-0.1839890 .596344-0.781359)_{\gamma} \\
& \text { Shape deformation matrix }(\gamma \mathrm{P} \gamma) \quad\left(\begin{array}{ccc}
0.028064 & 0.909040 & 0.119180 \\
0.029429 & -0.095386 & 1.124979
\end{array}\right) \\
& \text { Coordinate transformation matrix }(\gamma \mathrm{J} \alpha) \quad\left(\begin{array}{cccc}
0.579356 & 0.542586 & 0.102537 \\
0.014470 & 0.133650 & -0.788984 \\
-0.552000 & 0.572979 & 0.086936
\end{array}\right)
\end{aligned}
$$

The matrix notation used above is due to [6]. This set implies that

$$
\begin{gathered}
\left(\begin{array}{lll}
1 & \overline{1} & 1
\end{array}\right)_{\gamma}=\left(\begin{array}{lll}
0.012886 & 0.981915 & 0.978457
\end{array}\right)_{\alpha} \\
{\left[\begin{array}{lll}
1 & 1 & 0
\end{array}\right]_{\gamma}=\left[\begin{array}{lll}
0.927033 & 1.055684 & -1.071623
\end{array}\right]_{\alpha}}
\end{gathered}
$$

Notice that $\left(\begin{array}{lll}1 & \overline{1} & 1\end{array}\right)_{\gamma}$ is approximately parallel to $\left(\begin{array}{lll}0 & 1 & 1\end{array}\right)_{\alpha}$ and $\left[\begin{array}{lll}1 & 1 & 0\end{array}\right]_{\gamma}$ is approximately parallel to $\left[\begin{array}{lll}1 & 1 & 1\end{array}\right]_{\alpha}$. The actual relationship is irrational to ensure the invariant-line necessary for martensitic transformation.

The full twenty four crystallographic sets corresponding to the 24 variants of martensite possible in each austenite grain can be generated using symmetry operations.

It is necessary to replicate the observed austenite texture in order to model the transformation texture. The $\gamma$-textures of interest here are the Goss and Cube varieties [1]. Having chosen a sample reference frame in which the orientation of the tensile axis is one of the three axes, the first austenite grain is introduced in the exact required orientation (Goss or Cube). Another 499 grains are then generated by randomly choosing rotation axes, but limiting the right-handed angle of rotation to the range $0-45^{\circ}$. Allowing this angle to be any value would simply generate a random set of austenite grains. The results, in the form of $100_{\gamma}$ pole figures, are illustrated in Fig. 1.

Gey et al. proved that the transformation texture, beginning with the Goss and Cube austenite textures cannot be explained by allowing all martensite variants to form. In other words, something favours the formation of specific variants in each austenite grain. We assume here that it is the interaction of the applied stress with the shape deformation of the martensite which determines the variants that are favoured.

The interaction energy $U$ between the stress and the plate of martensite is given by [13]:

$$
U=\sigma_{N} \zeta+\tau s
$$




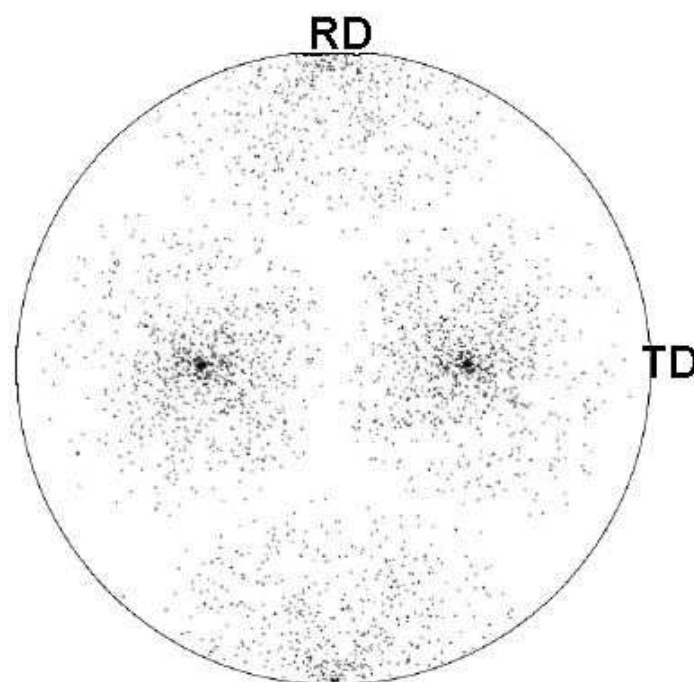

(a)

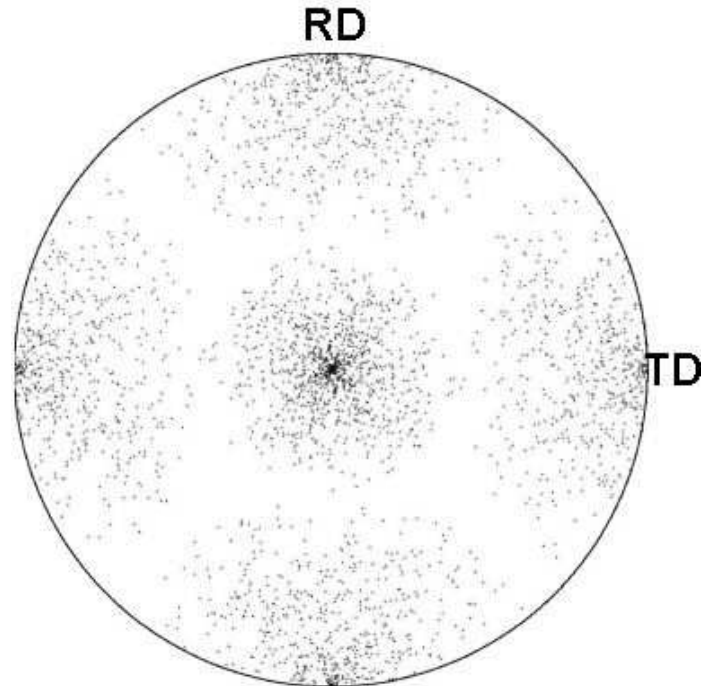

(b)

Fig. 1. $100_{\gamma}$ pole figure of a biased austenite. (a) Goss (b) Cube.

where $\sigma_{N}$ is the component of stress that is normal to the habit plane and $\tau$ the corresponding shear stress resolved on the habit plane, in the direction of the martensite shear. $\zeta$ and $s$ are the dilatational and shear strains due to the shape deformation of martensite, which can be shown using $(\gamma \mathrm{P} \gamma)$ to be 0.02536 and 0.2245 respectively [9] . Notice that calculating the interaction energy in this way is complete [13] compared with the approach in which only the symmetrical part of the shape deformation is used [5, 14].

A variant is said to be favoured when $U$ adds to the driving force for transformation. Each austenite grain is then allowed to transform to only the favoured variants, thus allowing the transformation texture to be calculated. It is emphasised that each austenite grain is transformed equally into the selected variants having equal fractions of each favoured variant. This is because the relationship between the interaction energy and fraction transformed is not clear. Therefore, although the crystallography should be correctly predicted, the detailed intensities may not be accurate.

For the Cube component of austenite texture, Fig. 2a shows the measured pole figure for martensite [1], Fig. 2b the calculated pole figure allowing only the favoured variants, and finally, Fig. 2c illustrates what should happen if all possible variants are allowed to form. Similar results for the Goss oriented austenite grains are shown in Fig. 3. The agreement between the calculated transformation texture based on favoured variants and the measured data is remarkably good.

It has been possible to reproduce the transformation texture that develops in particular components of the austenite texture, by allowing only those martensite variants which interact favourably with the applied stress to grow. The method is based on a self-consistent crystallographic set to define each martensite plate, and the interaction of the plate with the applied stress.

One outcome is that the stress alone seems sufficient to explain variant selection, even though the plastic strain in the original experiments reached some $10 \%$ elongation.

With respect to transformation textures, the method used here highlights the need for a complete set of crystallographic data for 18/8 stainless steels. 


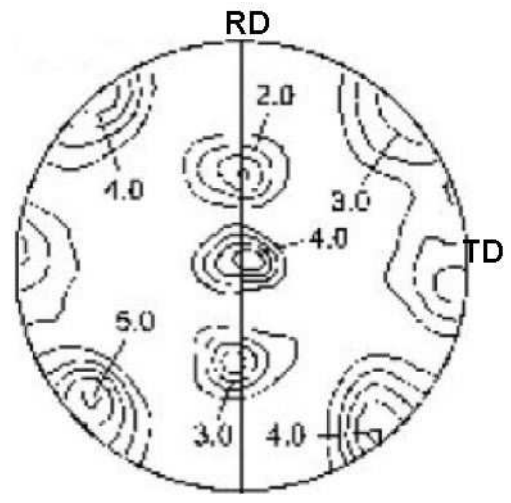

(a)

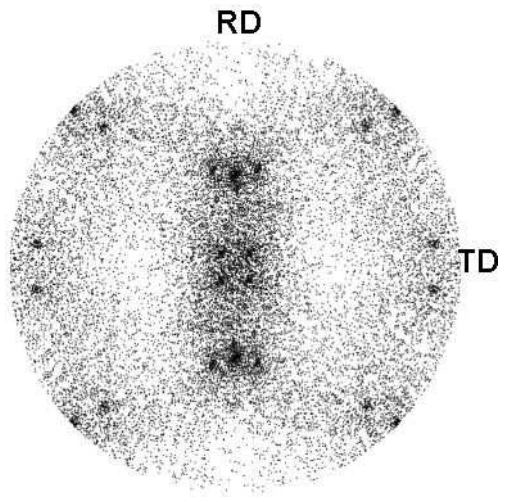

(b)

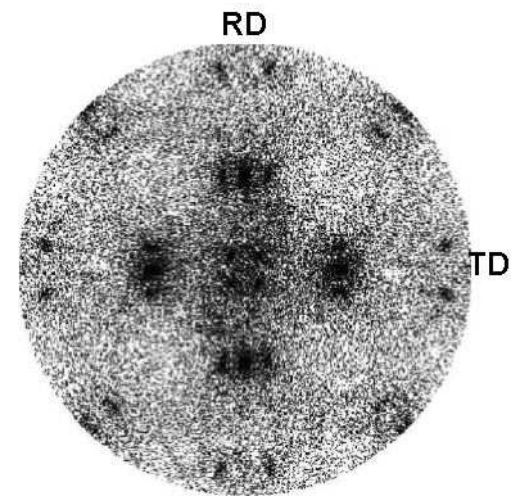

(c)

Fig. 2. $100_{\alpha^{\prime}}$ pole figures for transformation of Cube oriented austenite grains. (a) Experimental data [1]. (b) Calculations allowing only favoured variants of martensite to form in each austenite grain. (c) Calculations allowing all possible variants of martensite to form in each austenite grain.

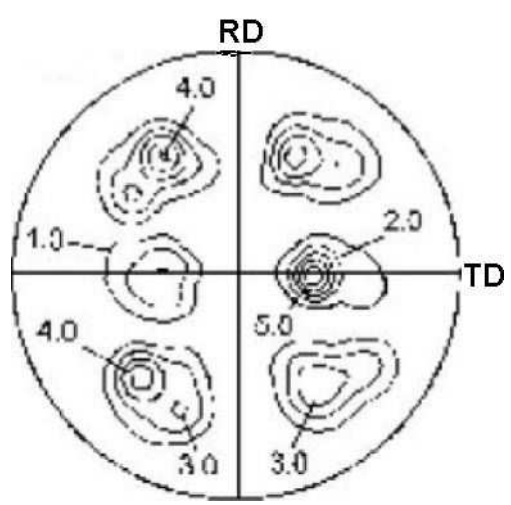

(a)

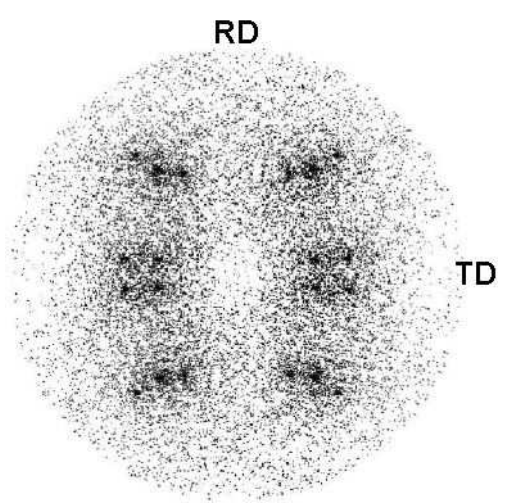

(b)

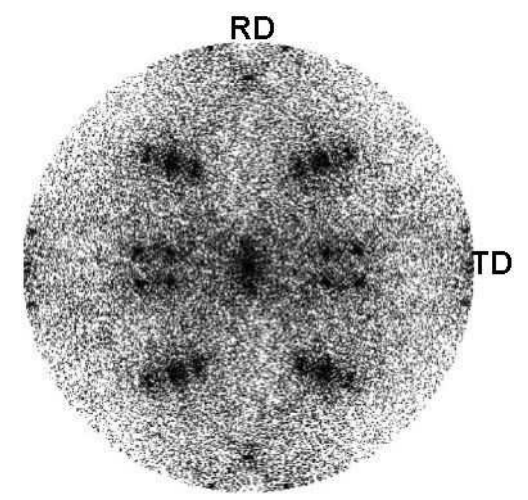

(c)

Fig. 3. $100_{\alpha^{\prime}}$ pole figures for transformation of Goss oriented austenite grains. (a) Experimental data [1]. (b) Calculations allowing only favoured variants of martensite to form in each austenite grain. (c) Calculations allowing all possible variants of martensite to form in each austenite grain.

The authors are grateful to the EPSRC (U.K.) and TATA Steel in India for funding this research.

\section{References}

[1] N. Gey, B. Petit, and M. Humbert. Metallurgical \& Materials Transactions, 36:3291-3299, 2005.

[2] L. Kestens, R. Decocker, and R. Petro. Materials Science Forum, 408-412:1173-1178, 2002.

[3] R. Petrov L. Kestens and Y. Houbaert. ISIJ International, 43:1444-1452, 2003.

[4] B. Brückner and G. Gottstein. ISIJ International, 41:468-477, 2001.

[5] P. Bate and B. Hutchinson. Acta Materialia, 48:3183-3192, 2000.

[6] J. S. Bowles and J. K. MacKenzie. Acta Metallurgica, 2:129-137, 1954.

[7] J. K. MacKenzie and J. S. Bowles. Acta Metallurgica, 2:138-147, 1954.

[8] M. S. Wechsler, D. S. Lieberman, and T. A. Read. Trans. AIME Journal of Metals, 197:15031515, 1953. 
[9] H. K. D. H. Bhadeshia. Geometry of Crystals. 2nd edition, Institute of Materials, 2001.

[10] G. Nolze. Zietschrift für Metallkunde, 95:744-755, 2004.

[11] R. Lagneborg. Acta Metallurgica, 12:823-843, 1964.

[12] D. J. Dyson and B. Holmes. Journal of the Iron and Steel Institute, 208:469-474, 1970.

[13] J. R. Patel and M. Cohen. Acta Metallurgica, 1:531-538, 1953.

[14] M. Humbert, B. Gardiola, C. Esling, G. Flemming, and K.E. Hensger. Materials Science Forum, 408:445-450, 2002. 